\title{
Desenvolvimento pós-embrionário de Pattonella intermutans (Thomson) (Diptera: Sarcophagidae) em diferentes dietas
}

\author{
Marcio S. Loureiro ${ }^{1,2}$, Vanderleia C. Oliveira ${ }^{1,2} \&$ José Mário d'Almeida ${ }^{1,3}$
}

\author{
${ }^{1}$ Laboratório de Biologia e Controle de Insetos Vetores, Instituto Oswaldo Cruz. Avenida Brasil 4365, 21045-900 Rio de Janeiro-RJ, \\ Brasil. vcris@ioc.fiocruz.br \\ ${ }^{2}$ Bolsista CNPq \\ ${ }^{3}$ Departamento de Biologia Geral, Universidade Federal Fluminense, Outeiro de São João Batista s/nº , 24020-150 Niterói-RJ, Brasil.
}

\begin{abstract}
Post-embryonary development of Pattonella intermutans (Thomson, 1869) in different diets. The purpose of this study was to evaluate the post-embryonary development of Pattonella intermutans (Thomson, 1969) in artificial diets composed of agar-agar. Diet $\mathrm{D}_{1}$ : whole dried milk + dried brewer's yeast; Diet $\mathrm{D}_{2}$ : whole dried milk + dried brewer's yeast + casein; Diet $\mathrm{D}_{3}$ : whole dried milk + whole egg; Diet $\mathrm{D}_{4}$ : bovine meal (control diet). The bovine meat was the best diet (larval weight $195.63 \mathrm{mg}$ and viability from larvae to adult $86,5 \%$ ), when compared to all other artificial diets. The following performances were obtained for experimental groups: diet $\mathrm{D}_{3}: 180.15 \mathrm{mg}$ and $63.5 \%$; diet $\mathrm{D}_{2}: 141.07 \mathrm{mg}$ and $61 \%$ and diet $\mathrm{D}_{1}: 147.98 \mathrm{mg}$ e $51.5 \%$.
\end{abstract}

Keywords. artificial diet; Pattonella intermutans; post-embryonic development; Sarcophagidae

\begin{abstract}
Resumo. Desenvolvimento pós-embrionário de Pattonella intermutans (Thomson) (Diptera: Sarcophagidae) em diferentes dietas. A proposta deste estudo foi de avaliar o desenvolvimento pós-embrionário de Pattonella intermutans (Thomson, 1869) em dietas artificiais preparadas com agar-agar. Dieta $\mathrm{D}_{1}$ : leite em pó integral + fermento biológico; Dieta $\mathrm{D}_{2}$ : leite em pó integral + fermento biológico + caseína; Dieta $\mathrm{D}_{3}$ : leite em pó integral + ovo cru; Dieta $\mathrm{D}_{4}$ : carne bovina moída (dieta controle). A carne bovina moída foi a dieta mais eficiente (peso larval de 195,63 mg e viabilidade de neolarva a adulto de $86,5 \%$ ), quando comparada com as dietas artificiais. Os seguintes resultados foram obtidos para o grupo experimental: Dieta $\mathrm{D}_{3}: 180,15 \mathrm{mg}$ e $63,5 \%$; Dieta $\mathrm{D}_{2}: 141,07 \mathrm{mg}$ e $61 \%$ e na Dieta $\mathrm{D}_{1}: 147,98 \mathrm{mg}$ e $51,5 \%$.
\end{abstract}

Palavras-Chave. Dieta artificial; Desenvolvimento pós-embrionário; Pattonella intermutans; Sarcophagidae.

A criação de insetos em dietas artificiais tem aplicações tanto na pesquisa básica quanto na aplicada (Parra 1990). São encontradas na literatura dietas artificiais para mais de 1.300 espécies de insetos, dentre elas, 279 se referem aos dípteros, sendo a maior parte de importância agrícola (Singh \& Moore 1985). Parra (1990) relatou que as principais vantagens do uso de dietas artificiais são a uniformidade nutricional do meio de cultura; maior uniformização biológica da colônia; a possibilidade de manter a colônia de maneira contínua; utilização das colônias em estudos de exigências nutricionais e menor risco de contaminação com patogénos. Zuben (1995) ressaltou que a ausência do odor de putrefação na criação de moscas em meios artificiais, é uma das vantagens que deve ser destacada.

Apesar das vantagens da manutenção das colônias em dietas artificiais, os trabalhos com dípteros muscóides são escassos, dentre eles pode-se destacar os de Lopes (1973), Leal et al. (1982), Zuben (1995) e D'Almeida \& Oliveira (2002).

Pattonella intermutans (Thomson, 1869) é um sarcofagídeo encontrado nas Américas (Lopes 1969), cujas larvas são tipicamente necrófagas desenvolvendo-se em carcaças de animais. Esta espécie foi considerada por Linhares (1981) em Campinas, D'Almeida (1984) no Rio de Janeiro e Dias et al. (1984) em Belo Horizonte, como uma espécie hemissinantrópica. Salviano (1996) ressaltou a importância desse sarcofagídeo para Entomologia Forense no Estado do Rio de Janeiro.
O presente estudo teve como objetivo avaliar a eficiência de diferentes dietas artificiais no desenvolvimento pósembrionário de $P$. intermutans.

\section{MATERIAL E MÉTODOS}

Colônias de P. intermutans foram estabelecidas no Laboratório de Biologia e Controle de Insetos Vetores, a partir de adultos capturados no campus da Fundação Oswaldo Cruz no Rio de Janeiro, RJ.

As dietas artificiais testadas foram modificadas a partir das utilizadas por Leal et al. (1982), para Chrysomya putoria (Wiedemann, 1830), e composta pelos seguintes ingredientes e proporções: Dieta $D_{1}$ : leite em pó integral $(20 \mathrm{~g})+$ fermento biológico (20 g) + agar-agar (2 g) + água destilada (200 ml); Dieta $\mathrm{D}_{2}$ : leite em pó integral $(20 \mathrm{~g})+$ fermento biológico $(20 \mathrm{~g})$ + caseína (1 g) + agar-agar (2 g) + água destilada (200 ml); Dieta $\mathrm{D}_{3}$ : leite em pó integral $(20 \mathrm{~g})+$ ovo cru (clara e gema) $(20$ g) + agar-agar $(2 \mathrm{~g})+$ água destilada $(200 \mathrm{ml}) ;$ Dieta $\mathrm{D}_{4}$ :carne bovina moída (100 g), utilizada como controle.

Para a manutenção das colônias e obtenção das neolarvas de $P$. intermutans utilizou-se a metodologia descrita por Oliveira et al. (2002). Em cada repetição (4) foram inoculadas 25 neolarvas em $25 \mathrm{~g}$ de dieta, perfazendo o total de 200 larvas/ dieta. Os potes de dietas contendo larvas foram mantidos em câmara climatizada regulada a $27 \pm 2^{\circ} \mathrm{C}, 60 \pm 10 \%$ U.R.

Após o abandono espontâneo das larvas maduras das 


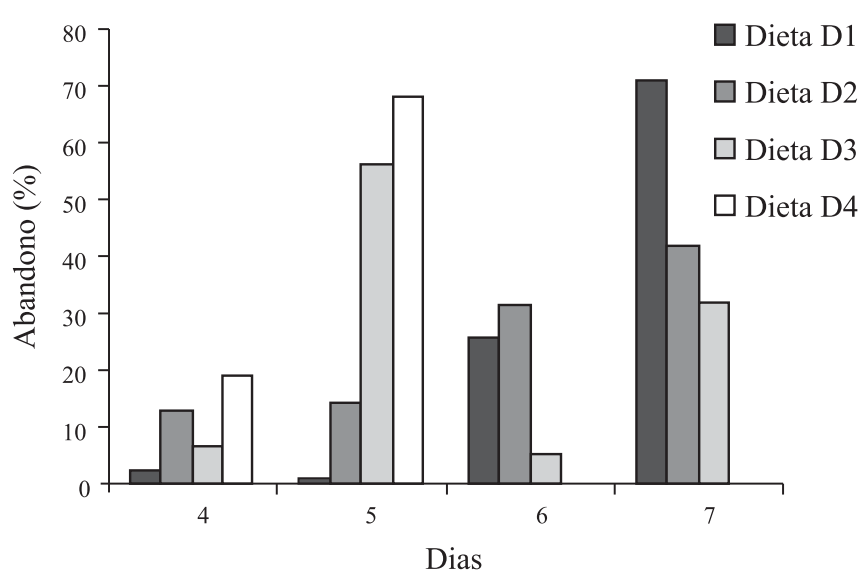

Fig. 1. Ritmo de abandono das larvas de Pattonella intermutans criadas em diferentes dietas, sob condições de laboratório.

dietas, estas foram pesadas individualmente e transferidas para tubos de ensaio contendo serragem, tampados com algodão e mantidos em câmara climatizada a $27 \pm 2^{\circ} \mathrm{C}, 60 \pm 10 \%$ U.R., até a emergência dos adultos.

Para avaliar a eficiência das dietas foram usados os seguintes parâmetros: 1)peso da larva madura; 2) duração média e a viabilidade dos estágios larval, pupal e do período de neolarva a adulto; 3 ) ritmo de abandono das larvas maduras e 4) ritmo de emergência de machos e fêmeas.

Os resultados foram submetidos à análise de variância (Anova) e as médias comparadas pelo teste de Tukey $(\propto=0,05)$.

\section{RESULTADOS EDISCUSSÃO}

Na maioria dos parâmetros biológicos analisados, a dieta controle, a base de carne, foi a mais eficiente, o que era esperado, pois as moscas deste gênero, em condições naturais, se desenvolvem em carcaças de animais (Lopes 1969). O mesmo foi observado por Leal et al. (1982) e D'Almeida et al. (2000) quando compararam a eficiência de dietas artificiais com carcaça

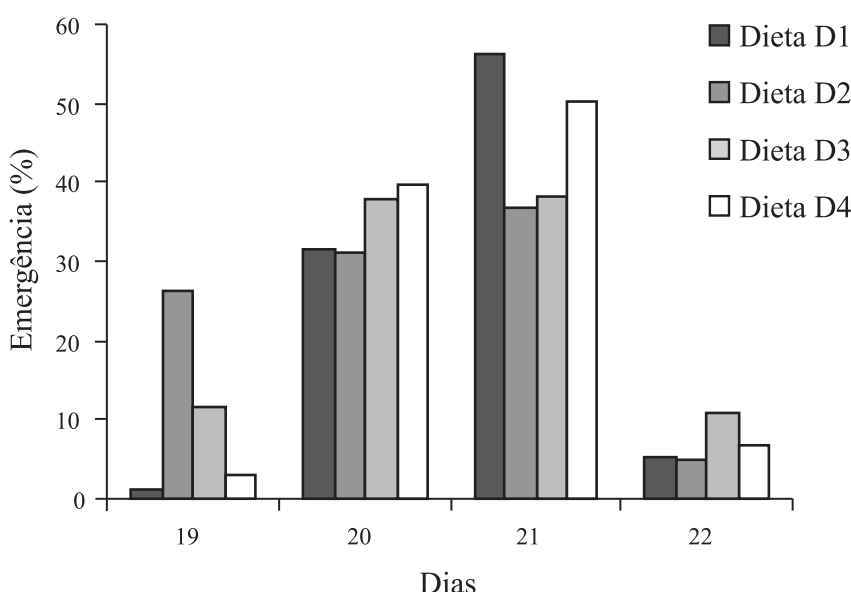

Fig. 2. Ritmo de emergência de Pattonella intermutans criadas em diferentes dietas, sob condições de laboratório.

de camundongo e carne putrefata para dois califorídeos, também com larvas necrófagas: Chrysomya putoria (Wiedemann, 1830) e C. megacephala (Fabricius, 1794), respectivamente.

A duração do estágio larval foi significativamente menor na dieta de carne e na dieta $\mathrm{D}_{3}$, não ocorrendo diferenças significativas entre elas (Tabela I). No entanto, a maior viabilidade larval foi na dieta de carne (Tabela II).

As larvas alimentadas com carne foram significativamente mais pesadas do que as das demais dietas o que, segundo Lopes (1941), resulta em adultos maiores. Entre as dietas $D_{1}$ e $\mathrm{D}_{2}$ não foram observadas diferenças significativas nos pesos das larvas (Tabela I). Segundo Lopes (1941), o desenvolvimento ovariano e a fecundidade dos adultos estão associados com o tamanho das larvas maduras, o que depende da qualidade e quantidade do alimento ingerido. No presente estudo, pode-se observar que o desenvolvimento das larvas de $P$. intermutans nas dietas artificiais $\mathrm{D}_{1}$ e $\mathrm{D}_{2}$, contrastou bastante quando comparado com as dietas $\mathrm{D}_{3}$ e $\mathrm{D}_{4}$, sugerindo que os ingredientes das dietas podem ter sido insuficientes.

Tabela I. Peso de larvas maduras e duração dos estágios larval, pupal e do período de neolarva a adulto de Pattonella intermutans, criadas em diferentes dietas, sob condições de laboratório.

\begin{tabular}{|c|c|c|c|c|}
\hline $\begin{array}{c}\text { Características } \\
\text { Biológicas }\end{array}$ & 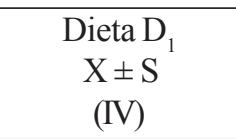 & $\begin{array}{c}\text { Dieta } \mathrm{D}_{2} \\
\mathrm{X} \pm \mathrm{S} \\
\text { (IV) }\end{array}$ & $\begin{array}{c}\text { Dieta } \mathrm{D}_{3} \\
\mathrm{X} \pm \mathrm{S} \\
\text { (IV) }\end{array}$ & $\begin{array}{c}\text { Dieta } \mathrm{D}_{4} \\
\mathrm{X} \pm \mathrm{S} \\
\text { (IV) }\end{array}$ \\
\hline Peso (mg) & $\begin{array}{c}147.98 \pm 38,20 \mathrm{a} \\
(47-220)\end{array}$ & $\begin{array}{c}141.07 \pm 27,94 \mathrm{a} \\
(32-220)\end{array}$ & $\begin{array}{c}180.15 \pm 26,87 \mathrm{~b} \\
(49-238)\end{array}$ & $\begin{array}{c}195.63 \pm 16,80 \mathrm{c} \\
(46-226)\end{array}$ \\
\hline Período larval(dias) & $\begin{array}{c}6.65 \pm 0,63 \mathrm{a} \\
(4-7)\end{array}$ & $\begin{array}{l}6.02 \pm 1,04 b \\
\quad(4-7)\end{array}$ & $\begin{array}{l}5.62 \pm 1,01 \mathrm{c} \\
\quad(4-7)\end{array}$ & $\begin{array}{l}5.76 \pm 0,43 c \\
\quad(5-6)\end{array}$ \\
\hline Período pupal(dias) & $\begin{array}{c}14.02 \pm 0,54 \mathrm{a} \\
(13-15)\end{array}$ & $\begin{array}{c}14.07 \pm 0,60 \mathrm{a} \\
\quad(13-16)\end{array}$ & $\begin{array}{l}14.87 \pm 0,84 \mathrm{~b} \\
\quad(14-17)\end{array}$ & $\begin{array}{l}13.87 \pm 0,51 \mathrm{a} \\
(13-15)\end{array}$ \\
\hline $\begin{array}{l}\text { Período de neolarva } \\
\text { a adulto (dias) }\end{array}$ & $\begin{array}{l}20.73 \pm 0,58 \mathrm{a} \\
\quad(19-22)\end{array}$ & $\begin{array}{l}20.16 \pm 0,85 \mathrm{~b} \\
\quad(18-22)\end{array}$ & $\begin{array}{l}20.50 \pm 0,85 \mathrm{a} \\
\quad(19-22)\end{array}$ & $\begin{array}{l}18.65 \pm 0,64 \mathrm{c} \\
\quad(17-20)\end{array}$ \\
\hline
\end{tabular}

X: Média; S: Desvio Padrão; IV: Intervalo de Variância

* Médias com pelo menos uma letra em comum não diferem entre si, e as seguidas de letras diferentes, diferem significativamente $(\propto=0,05)$, pelo teste de comparações múltiplas de Tukey. 
Tabela II. Viabilidade dos estágios larval, pupal e do período de neolarva a adulto de Pattonella intermutans, criadas em diferentes dietas, sob condições de laboratório.

\begin{tabular}{ccccc}
\hline Viabilidade & $\begin{array}{c}\text { Dieta } \mathrm{D}_{1} \\
\%\end{array}$ & $\begin{array}{c}\text { Dieta } \mathrm{D}_{2} \\
\%\end{array}$ & $\begin{array}{c}\text { Dieta } \mathrm{D}_{3} \\
\%\end{array}$ & $\begin{array}{c}\text { Dieta } \mathrm{D}_{4} \\
\%\end{array}$ \\
\hline Larval & 60.5 & 67 & 67.5 & 99 \\
Pupal & 85.1 & 91 & 94 & 86.7 \\
Neolarva a adulto & 51.5 & 61 & 63.5 & 86.5 \\
\hline
\end{tabular}

No preparo das dietas utilizou-se o leite em pó integral, que contém em sua composição duas importantes proteínas, a caseína e a lactoalbumina, sendo que a primeira, é uma das mais utilizadas para o desenvolvimento dos insetos (Parra 1990). Segundo House (1961) uma dieta artificial completa deve conter: proteínas ou aminoácidos, carboidratos, lipídios, vitaminas, principalmente as do grupo B, sais minerais e água. Entretanto, o balanço entre os nutrientes nas dietas é fundamental para proporcionar um melhor desenvolvimento dos insetos (House 1969). Em relação a dieta $D_{3}$, associou-se ao leite em pó, ovo cru. Na composição da clara do ovo a principal proteína é a ovoalbumina que corresponde a $59 \%$ de toda massa do ovo e na gema encontram-se proteínas, vitaminas, ácidos graxos, fosfolípideos e minerais (Bobbio \& Bobbio 1992).

Na duração do estágio pupal, a dieta $\mathrm{D}_{3}$ foi a que apresentou o maior período comparado as demais dietas (Tabela I). A maior viabilidade pupal foi observada na dieta $\mathrm{D}_{3}$ (Tabela II).

$\mathrm{Na}$ avaliação da duração do período de neolarva a adulto a carne foi a que apresentou o menor período, sendo considerada a dieta mais viável, com $86,5 \%$. Segundo D'Almeida et al. (2000) a avaliação das dietas com base na duração e viabilidade do período de neolarva a adulto é mais eficiente, evitando as distorções existentes entre a duração dos estágios larval e pupal.

Na Fig. 1 pode-se observar o ritmo de abandono das larvas maduras das dietas. $\mathrm{Na}$ dieta $\mathrm{D}_{1}$, a maioria das larvas abandonou a dieta no $7^{\circ}$ dia, enquanto que as larvas da dieta $\mathrm{D}_{2}$ apresentaram um ritmo de abandono contínuo, tendo sido também observado um pico no $7^{\circ}$ dia. Na dieta $\mathrm{D}_{3}$ abandonaram em maior número no $5^{\circ}$ dia coincidindo com o pico de abandono da dieta $\mathrm{D}_{4}$, o que novamente aproxima a dieta de leite em pó integral e ovo $\left(\mathrm{D}_{3}\right)$ com o controle (carne).

$\mathrm{O}$ ritmo de emergência dos adultos das diferentes dietas teve inicio no $19^{\circ}$ dia, tendo sido observado um pico de emergência no $21^{\circ}$ dia para as dietas $D_{1}$ e $D_{4}$, enquanto que nas dietas $\mathrm{D}_{2} \mathrm{e} \mathrm{D}_{3}$ os adultos apresentaram pico de emergência no $20^{\circ} \mathrm{e} 21^{\circ}$ dias (Fig. 2).

$\mathrm{O}$ aumento do estágio larval e a diminuição do estágio pupal observado nas dietas artificiais $\mathrm{D}_{1}$ e $\mathrm{D}_{2}$ pode ser uma estratégia para compensar a queda na qualidade nutricional do alimento consumido (Ullyett 1950; Kamal 1958).

Considerando-se que as larvas de P. intermutans, em

Recebido em 06.05.2004; aceito em 20.09.2004 condições naturais, são necrófagas, a viabilidade obtida nas dietas artificiais pode ser melhorada, através de estudos que visem o melhor balanceamento nutricional das dietas, principalmente da Dieta $\mathrm{D}_{3}$, que apresentou viabilidade superior a $60 \%$, o que é promissor.

\section{REFERÊNCIAS}

Bobbio, F. O. \& P. A. Bobbio. 1992. Química no Processamento de Alimentos. 2 ed. São Paulo, Varela, 151 p.

d' Almeida, J. M. 1984. Sinantropia de Sarcophagidae (Diptera) na Região Metropolitana do Rio de Janeiro. Arquivos da Universidade Federal Rural do Rio de Janeiro 7: 101-110

d' Almeida, J. M.; M. B. Fraga \& C. L. Ferro. 2000. Desenvolvimento pós-embrionário de Chrysomya megacephala (Diptera: Calliphoridae), em dietas artificiais. Entomologia y Vectors 7: 155-162.

d' Almeida, J. M. \& V. C. Oliveira. 2002. Dietas artificiais para a criação, em laboratório, de Chrysomya (C. megacephala, C. albiceps e C. putoria) (Diptera: Calliphoridae). Entomologia y Vectores 9: 79-91.

Dias, E. S.; D. P. Neves \& H. S. Lopes. 1984. Estudos sobre a fauna de Sarcophagidae (Diptera) de Belo Horizonte, Minas Gerais. I. Levantamento taxonômico e sinantrópico. Memórias do Instituto Oswaldo Cruz 79: 83-91.

House, H. L. 1961. Insect nutrition. Annual Review of Entomology 6: $13-26$.

House, H. L. 1969. Effects of different proportions of nutrients on insects. Entomologia Experimentalis et Applicatta 12: 651669.

Kamal, A. S. 1958. Comparative study of thirteen species of sarcosaprophagous calliphorids and sarcophagids. Annals of the Entomological Society of America 51: 261-271.

Leal, T. T.; A. P. Prado \& J. A. Antunes. 1982. Rearing the larvae of the blowfly Chrysomya cloropyga (Wiedemann) (Diptera: Calliphoridae) on oligidic diets. Revista Brasileira de Zoologia 1: $41-44$.

Linhares, A. X. 1981. Sinantropy of Calliphoridae and Sarcophagidae (Diptera) in the city of Campinas, São Paulo, Brazil. Revista Brasileira de Entomologia 25: 189-215.

Lopes, H. S. 1941. Esqueleto céfalo-faringeano e a sua importância na classificação. Revista Brasileira de Biologia 1: 215-221.

Lopes, H. S. 1969. A Catalogue of the Diptera of the Americas South of the United States: Family Sarcophagidae. Departamento de Zoologia, Secretaria da Agricultura, São Paulo, 103: $88 \mathrm{p}$.

Lopes, H. S. 1973. Collecting and rearing Sarcophagidae flies (Diptera) in Brazil during forthy years. Anais da Academia Brasileira de Ciência 45: 279-291.

Oliveira, V. C.; R. P. Mello \& R. F. S. Santos. 2002. Bionomics Aspects of Pattonella intermutans (Thomson, 1869) (Diptera, Sarcophagidae) under laboratory conditions. Brazilian Archives of Biology and Technology 45: 473-477.

Parra, J. R. P. 1990. Técnicas de Criação de Insetos para Programas de Controle Biológico. Piracicaba, ESALQ, 125 p.

Singh, P. \& R. F. Moore. 1985. Handbook of Insect Rearing. Elsevier Publ, $600 \mathrm{p}$.

Salviano, R. J. B. 1996. Sucessão de Diptera Caliptrata em carcaça de Sus scrofa Linnaeus, Rio de Janeiro, RJ, Brasil. Tese de Mestrado, Universidade Federal Rural do Rio de Janeiro, 158 p.

Ullyett, G. C. 1950. Competition for food and allied phenomena in sheep-blowfly populations. Philosophical Transactions of the Royal Society of London B, Biol. Sci., 234: 77-174

Zuben, C. J. 1995. Competição larval e efeitos sobre a dinâmica populacional de Chrysomya megacephala (F.) (Diptera: Calliphoridae). Tese de Doutorado, UNESP, Rio Claro, 132 p. 